\title{
Assessing the shelf life of aseptically prepared injectables in ready to administer containers
}

\author{
Mike Allwood
}

An important role for hospital pharmacy is to provide injectable drugs in ready to administer containers which are prepared in a pharmacy aseptic facility and supplied to wards either for individual patients or batch prepared as ward stock. The latter method is clearly far more efficient and less demanding on service provision and personnel, and is becoming therefore the favoured method. However, this requires that first, products are prepared under strict aseptic conditions, to remove the microbiological risks associated with the preparation of injections in uncontrolled environments and second, the ability to assign extended shelf lives to such pharmacy prepared products. Unfortunately, in general, pharmaceutical manufacturers are unable to help in this respect as they are constrained by the regulations concerning the information which can be included in SPCs for licensed products, normally limited to advice on storage for up to $24 \mathrm{~h}$ after opening the original container. This is based on the notion that microbiological risks must be contained and therefore this ' $24 \mathrm{~h}$ rule' is imposed. This does not reflect the chemical stability of the active drug in the product, which is normally, with a few exceptions, sufficiently stable to

Correspondence to Professor M Allwood, 16 Rectory Lane, Breadsall, Derby, DE21 5LL, UK; m.c.allwood@derby.ac.uk assign shelf lives measured in weeks rather than days. In fact, most injectable drugs are remarkably stable even after dilution and transfer to syringes or intravenous bags.

The responsibility for assigning shelf lives to hospital pharmacy prepared injections in ready to administer containers (syringes, intravenous bags, ambulatory device reservoirs) is entirely the hospital pharmacist's responsibility. So how can we insure we generate the evidence necessary to fulfil this requirement? The primary requirement is an assay method which fulfils all the requirements for accuracy, precision and sensitivity, as defined by many official sources.

However, there is an additional requirement if an analytical method is to be applied to this particular application. The method must be, and shown to be, stability indicating. Traditional methods, such as UV absorption will not meet this requirement. In most cases, a chromatographic method is the most favourable as it is normally able to separate drug from degradation products and excipients. Liquid chromatographic methods are also relatively simple to apply. Proofs are required that the method is truly stability indicating. The optimum approach would be to show that retention times of all possible degradation products are different from the drug compound. However, in reality, knowledge of all of the possible degradation products from any individual drug will not be known. So an acceptable compromise must be sort. It is generally acknowledged that forced degradation studies meet this requirement. Samples of the diluted drug product are stressed by heating in mild acid, alkali and oxidation conditions. Chromatographic evidence is then obtained to show reduction in drug peak, and that secondary peaks which represent degradation products, usually with shorter retention times, are detected. This is further supported by evidence that the purity of the drug is assured by, for example, UV diode array drug peak analysis.

The application of this approach to testing samples of product prepared in the hospital pharmacy and subject to storage conditions appropriate to the drug can then be used to justify extended shelf lives, remembering also that shelf life may also be governed by the nature of the container and of the specific commercial source of the drug product.

\section{Competing interests None.}

Provenance and peer review Commissioned; not externally peer reviewed.

Published Online First 22 March 2012

European Journal of Hospital Pharmacy 2012;19:277.

doi:10.1136/ejhpharm-2012-000092 